\title{
Outcomes, Complications and Management of the Congenital Aural Atresia Surgery
}

\author{
Oğuz Kuşcu1 ${ }^{*}$, Emel Tahir ${ }^{1}$, Önder Günaydın1, Ömer Faruk Ünal², Umut Akyol1 \\ ${ }^{1}$ Deparment of Otorinolaryngology, Head and Neck Surgery, Hacettepe University, Ankara, Turkey \\ ${ }^{2}$ Deparment of Otorinolaryngology, Head and Neck Surgery, Özel Acıbadem University, Istanbul, Turkey \\ Email: 마.kuscu@gmail.com, ofu@trnet.tr, uakyol@gmail.com
}

Received 4 April 2014; revised 3 May 2014; accepted 2 June 2014

Copyright (C) 2014 by authors and Scientific Research Publishing Inc.

This work is licensed under the Creative Commons Attribution International License (CC BY). http://creativecommons.org/licenses/by/4.0/

cc) (7) Open Access

\begin{abstract}
Congenital aural atresia is characterized by aplasia of the external auditory canal (EAC), often associated with abnormalities of the auricula (microtia), the middle ear, and, occasionally, the inner ear structures. The aim of this study is to evaluate the surgical challenges, complications and long term results of congenital aural atresia (CAA) surgery. Records of 103 patients who underwent surgery because of congenital aural atresia were reviewed between 1992 and 2012 in a tertiary referral center. Audiological and surgical events were observed. Surgical technique, incision type, graft material and long term results were noted. Postoperative visit records and audiograms were investigated. Many types of ossicular chain abnormalities such as absence of an ossicle, deformity of an ossicle or ossicular block were observed. Facial canal dehiscence or abnormal location of the facial nerve was seen in $\mathbf{3 3 . 9 \%}$ of patients. Closure of the air-bone gap (ABG) to $30 \mathrm{~dB}$ or less at short-term follow-up occurred in $\mathbf{6 3 . 1 \%}$ of patients. Major complications were as follows: restenosis of the external ear canal, hearing loss, facial paralysis, iatrogenic cholesteatoma and perforation of the graft. The air-bone gap cannot be totally closed in all aural atresias, and there is also the chance of sensorineural type hearing loss. Success of the surgery depends on accompanying abnormalities like unexpected route or dehiscence of facial nerve, status of the ossicular chain and middle ear cleft and surgical techniques. Long term results of congenital aural atresia surgery are encouraging and promising.
\end{abstract}

\section{Keywords}

Congenital Aural Atresia, Facial Canal Route, Bone Mass, Air-Bone Gap

\footnotetext{
${ }^{*}$ Corresponding author.
}

How to cite this paper: Kuşcu, O., Tahir, E., Günaydın, Ö., Ünal, Ö.F. and Akyol, U. (2014) Outcomes, Complications and Management of the Congenital Aural Atresia Surgery. International Journal of Otolaryngology and Head \& Neck Surgery, 3, 166-172. http://dx.doi.org/10.4236/ijohns.2014.34031 


\section{Introduction}

Congenital aural atresia is characterized by aplasia of the external auditory canal (EAC), often associated with abnormalities of the auricula (microtia), the middle ear, and, occasionally, the inner ear structures. EAC atresia is more commonly osseous than membranous, and osseous atresia usually coexists with malformation of the middle ear. Generally, more severe microtia indicates a more severe middle ear abnormality, although some atresias are associated with normal pinnae [1].

The main purpose of congenital aural atresia (CAA) surgery is to provide patients with serviceable hearing and a new external auditory canal [2]. Techniques of canalplasty, meatoplasty, tympanoplasty, and ossiculoplasty have improved considerably, but surgical correction of congenital aural atresia is still one of the most difficult and challenging procedures performed on the ear. What makes this a challenging surgery is the lack of landmarks, altered anatomy of the facial nerve and middle ear, limited space for middle ear reconstruction, and delayed healing process. These factors make it more difficult to achieve the primary goals of surgery, such as restoring hearing and the maintenance of a patent, reconstructed EAC that is free of infection. The complexity of this surgery requires a good knowledge of the surgical anatomy of the facial nerve, oval window, and inner ear, and their congenital variations [3].

CAA surgery is very difficult even in experienced hands, and there are many complications with this procedure. In this study, we retrospectively examined long-term results of 103 patients operated for CAA and discussed the difficulties encountered in this challenging surgical procedure.

\section{Materials and Methods}

\subsection{Patients}

We reviewed the medical records of 103 patients who were operated for CAA between 1992 and 2012 at the Department of Otolaryngology Head and Neck Surgery, Hacettepe University Medical Faculty. Medical records of the patients were evaluated for their age, sex, ENT examination results (Jahrsdoerfer score, and the specific point value, 0-1-2, assigned to each anatomical structure based on the Jahrsoderfer grading scale), surgical and audiological findings (Table 1). In 81 patients the anterior approach, in 21 patients the transmastoid approach and in one patient the combined approach was used. Facial nerve monitoring was used in all patients were operated after the late 90’s. Temporalis muscle fascia was used as a graft material for all patients. In a revised case, a perichondrium graft was used. To line the newly formed EAC, split thickness skin grafts harvested mostly from the lateral thigh and arm were used. In 6 patients tragus skin, and in 2 postauricular skin flap, was used to line the ear canal. Information was obtained from the patients' preoperative records, operative notes, audiograms, and postoperative office visits. All patients underwent audiometric testing before surgery and were typically seen approximately 3 months after surgery for a full audiometric follow-up, with many office visits again later.

Table 1. Jahrsdoerfer grading scale score.

\begin{tabular}{cc} 
Findings & Score \\
\hline Stapes bone & 2 \\
Oval window open & 1 \\
Middle ear space & 1 \\
Facial nerve & 1 \\
Malleus-incus complex & 1 \\
Mastoid pneumatization & 1 \\
Incus-stapes connection & 1 \\
Round window & 1 \\
External ear & 1 \\
Total possible score & 10 \\
\hline
\end{tabular}




\subsection{Surgical Technique}

After the incision, drilling begins at the level of the linea temporalis, just posterior to the glenoid fossa in an anteroposterior direction. The middle fossa tegmen is identified and followed to the epitympanum, where the ossicules are identified. Usually, the malleus and incus are found as a block mass. In most cases, the ossicular mass in the epitympanum is meticulously dissected free of the atretic bone and is left intact. The fibrous ligaments and bony adhesions are dissected with a thin pick. Drilling is continued to create a new ear canal measuring about 1.5 times the normal size, with care taken to not expose the temporomandibular joint space. If the anterior part of the new canal is not stable enough because of an opening to the joint, a cartilage or bone graft is used as a support.

Until the late 90s, we used a transmastoid approach. After this time our preference changed to the anterior approach, so that we do not expose the mastoid cells. We protect the external ear canal skin flap. After drilling the EAC we create a new and wider EAC. The middle fossa tegmen is identified and followed to the epitympanum, where the ossicules are identified. Temporalis fascia is used as a graft for the formation of a new tympanic membrane; a split thickness skin graft and the patient's own skin flap, together, are graft materials for the EAC. After the skin graft is used to line the new ear canal lying over the fascia, antibiotic-soaked Gelfoams are used to fill the canal. Skin, subcutaneous tissue, and cartilage are removed to create the new meatus. The lateral edge of the skin graft is sutured to the meatal skin and a wick tamponade is then placed in the outer portion of the EAC and the meatus.

\subsection{Data Analysis}

In our study, pure-tone air- and bone-conduction thresholds were obtained before and after the surgery, with thresholds at 500,1000, 2000, and $4000 \mathrm{~Hz}$ used to calculate the pure tone averages. Air and bone from the same test were used to calculate the air bone gap (ABG). A successful hearing result is determined as a postoperative ABG of $30 \mathrm{~dB}$ or less. For continuous variables, independent groups were compared using t-tests while paired-comparisons were made using paired t-tests. The criterion for statistical significance was set at $\mathrm{p}<$ 0.05 .

\section{Result}

All patients included in the study were score 6 - 8 on the basis of their preoperative Jahrsoderfer grading score. There were 60 males and 43 females, with ages ranging between 4 and 34 years, with a mean age of 14.92 patients had unilateral, while 11 had bilateral atresia. The duration of follow-up ranged from 16 to 90 months, with a mean duration of 53 months. The malleus was absent in 19 (18.4\%) cases and appeared normal in 49 (47.5\%) cases. The incus was absent in 27 (26.2\%) cases and had a normal appearance in 41 (39.8\%) cases. In the other $33(32 \%)$ cases, the malleus and incus were present as a ossicular mass, fixed and attached to the atretic bone (Figure 1). The stapes was normal in $97(94.1 \%)$ cases and was deformed in $4(3.8 \%)$ cases $(p<0.05)$. In no case was the stapes footplate absent or fixed. In 50 (48.5\%) patients, the graft was laid on the malleus, keeping the ossicular chain intact, whereas in 39 (37.8\%) patients the malleus-incus block was removed and the graft was laid on the stapes. In 4 (3.8\%) cases the incus and stapes, 5 (4.8\%) cases the malleus and incus, 2 (1.9\%) cases a cartilage graft and 1 case a cortical bone graft were used to form the ossicular chain. In $2(1.9 \%)$ of the patients, the middle ear cleft was not found. The facial canal was dehiscent in 29 (28.1\%) cases and the facial nerve followed an unexpected route in 6 (5.8\%) patients. Accompanying abnormalities are summarized in Table 2.

In preoperative PTAs (pure tone audiograms), the average bone threshold was found to be $34.1 \mathrm{~dB}$. The postoperative average bone threshold was found to be $31.7 \mathrm{~dB}$ and the average air threshold was $59.3 \mathrm{~dB}$, with a postoperative average ABG of $27.6 \mathrm{~dB}$ (Figure 2). According to the postoperative 3rd month audiogram, 65 (63.1\%) patients’ ABG was under $30 \mathrm{~dB}$.

Postoperative complications included restenosis, total hearing loss, temporary facial nerve paralysis, iatrogenic cholesteatoma formation in the middle ear and graft perforations.Complications are summarized in Table 3.

\section{Discussion}

Surgery for congenital aural atresia is postponed until 5 - 7 years age, for the better cooperation of patients with 
Table 2. Accompanying abnormalities.

\begin{tabular}{cc}
\hline Abnormality & Number of patients \\
\hline Ossicular block & $33(32 \%)$ \\
Dehiscent facial canal & $29(28.1 \%)$ \\
Absence of incus & $27(26.2 \%)$ \\
Absence of malleus & $19(18.4 \%)$ \\
Deformity of stapes & $4(3.8 \%)$ \\
\hline
\end{tabular}

Table 3. Postoperative complications.

\begin{tabular}{cc}
\hline Complication & Number of patients \\
\hline Restenosis of the EAC & $15(14.5 \%)$ \\
Temporary facial paralysis & $3(2.9 \%)$ \\
Graft perforation & $2(1.94 \%)$ \\
İatrogenic cholesteatoma & 1 \\
Total hearing loss & 1 \\
\hline
\end{tabular}

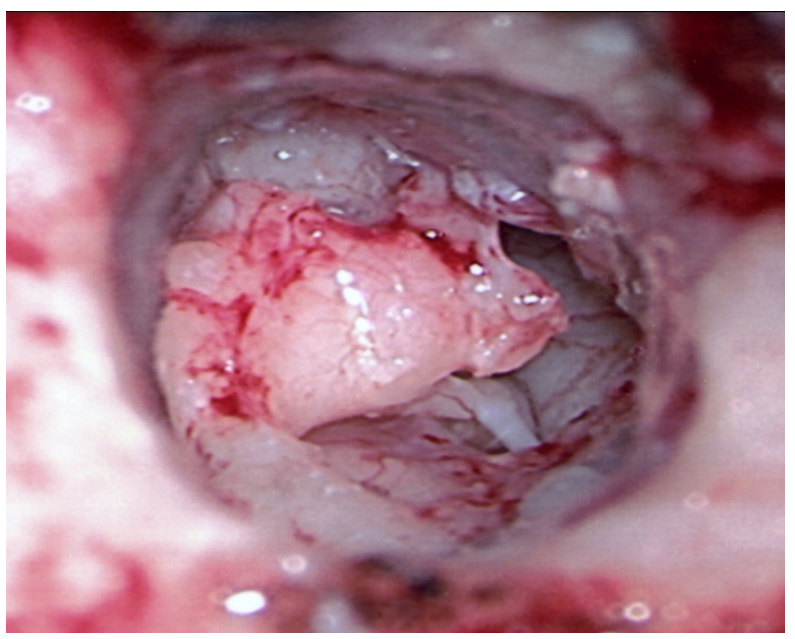

Figure 1. Malleus-incus atretic bone.

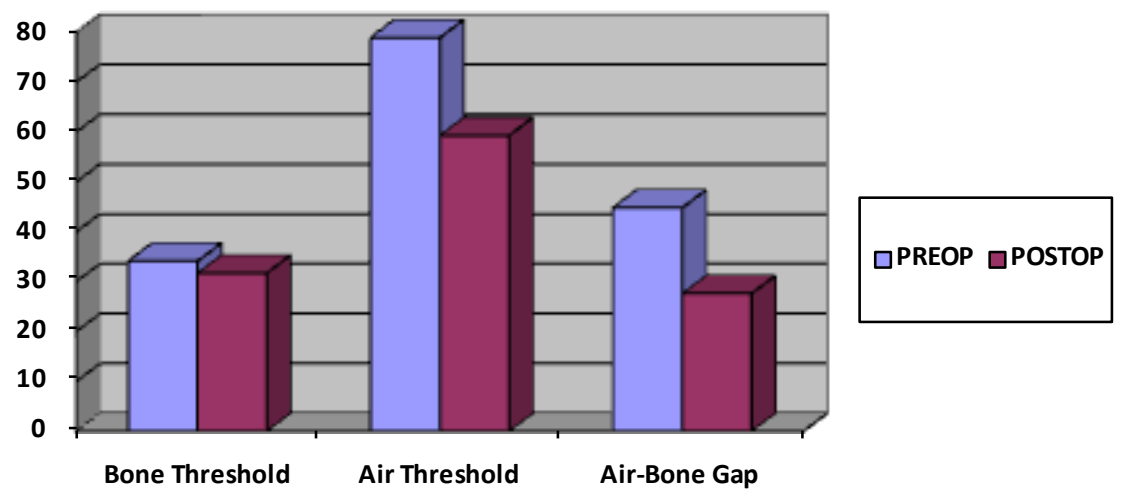

Figure 2. Audiologic result.

audiometric evaluation and completion of mastoid pneumatization, unless there is a cholesteatoma, infected fistula or facial paralysis [4]. In unilateral atresia, surgery can be postponed until the patient is mature enough to decide for himself. Bilateral atresia usually requires surgery to restore hearing. Before surgery as soon as possible patients should be fitted with appropriate amplification. Surgical intervention in bilateral cases followed with 
appropriate hearing stimulation was generally performed around age 5 - 7 years [5]. We should always keep in mind that there might be a total hearing loss, so that surgery on the only hearing ear is contraindicated. If there is a sclerotic mastoid, surgery for aural atresia is challenging, since there is less mastoid aeration, and formation of an ear canal is more difficult [6]. Difficulties encountered during the surgical correction of congenital aural atresia are as follows [7].

\subsection{Problems with Incisions}

Both an anterior and posterior approach can be used. Usually the anterior approach is preferred by the senior author. An endaural incision is modified to form an S shape or to create a superiorly based skin flap. To decide the place of incision, the root of the zygomatic bone and the mandibular condyle, anterior border of the mastoid and mastoid tip should be identified [8]. The EAC should be constructed on the same plane as the contralateral EAC. In patients where there is hemifacial hypoplasia or in patients with bilateral aural atresia, it is difficult to choose an incision site. In our study, 15 (14.5\%) patients with bilateral aural atresia developed restenosis because of incision problems. For that reason either a horizontal plane passing between the upper border of the tragus to the inferior orbital ridge, or a horizontal line passing through the midline between the nasion and subnasal point, is used to locate the EAC.

\subsection{Problems Concerning the Approach to the Middle Ear Cleft}

Either transmastoid or anterior approaches are used to enter the middle ear cleft. In the anterior approach, drilling of the EAC is done between the temporal line and the temporomandibular joint [9]. If the temporomandibular joint is entered during drilling, the defect should be covered with a cartilage graft and the fascia covering it. Otherwise the mandibular condyle may protrude into the ear canal. In the transmastoid approach, the middle cranial fossa duramater is the pointer to the epitympanium. The EAC should be drilled twice as wide as normal, which can cause facial nerve problem [10]. In our study we observed that $28.1 \%$ of patients had facial canal dehiscence, while $2.9 \%$ of patients had temporary facial paralysis. On the other hand, if the mastoid cavity is drilled too large there might be postoperative problems such as frequent infection and debris collection.

\subsection{Problems with the Removal of the Atresia Plate}

The atresia plate is drilled with a diamond burr or with a curette until the ossicles are exposed. Since the ossicles are attached to the medial side of the atresia plate, the surgeon should be very careful not to luxate the ossicular chain [8] [9] [11]. The medial periosteum of the atresi plate should be preserved, but this is difficult to do since it is closely adherent, and the mucoperiosteum may be damaged during elevation. After removal of the atresia plate, bone around the ossicles is drilled to enlarge the entrance to the middle ear cleft.

\subsection{Problems Concerning the Middle Ear Cleft}

We should first decide whether the space entered is the tympanic cavity or a mastoid cell. In the tympanic cavity we should see ossicles, either complete or deformed. The mucosal covering of the tympanic cavity is thicker than the mucosa of the mastoid cells, which is also helpful to identify the location. Before the operation, CT or MRI are very helpful to gain information about the status of the ossicles and middle ear.

\subsection{Ossicular Problems}

If ossicles are mobile and functional they should be preserved. In our patient population the most common ossicular finding was a fused malleus-incus and a normal stapes [12]. If there is a fused and nonfunctioning malleus and incus it should be removed. In our study we found that $37.8 \%$ of patients had malleus-incus blocks and we removed them. The graft was then laid on the mobile stapes or footplate. To determine the ossicular status, the tympanic cavity should be widely exposed to visualize the oval and round windows; to do this, the inferior part of the atresia plate is removed with a curette.

\subsection{Problems Concerning the Facial Nerve}

It should always be kept in mind that the facial nerve may follow an unexpected route. The degree of microtia is 
parallel to the anomalous route of the facial nerve [13]. In our study, we observed that $28.1 \%$ of patients had facial canal dehiscence, while $2.9 \%$ of patients had temporary facial paralysis. In severe anomalies the vertical segment of the facial nerve is located anteriorly. With sclerotic mastoid problems, damage to the facial nerve is more possible [14]. At the posterioinferior part of the atresia plate the facial nerve may be confused with the annulus fibrosus [14]. When bone is covering the facial nerve, it is identified with the help of the vessels on the nerve sheath. If the middle ear cleft is narrow, the probability of damaging the facial nerve is higher [15]. The semicanal for the tensor tympani muscle is useful in identifying the facial canal. This structure is continuous with the upper border of the oval window and with the lower border of the facial canal inferiorly [14] [16]. The tympanic nerve on the promontorium is located vertically, and when followed superiorly points to the cochleariform process, which is located just anterior to the facial canal [14] [17].

\subsection{Problems with Hearing}

The air-bone gap cannot be closed totally in all aural atresias, and there is also a chance of sensorineural type hearing loss. In our patient group, the postoperative ABG was under $30 \mathrm{~dB}$ in 65 (63.1\%) patients. We should construct a vibrating ear drum and a wide enough EAC.

\subsection{The Following Factors Effect Hearing Results}

1) Ossicles: If the stapes is fixed it should not be removed in the first operation. In our study there were no patient with fixation of the stapes. If the malleus and incus are fixed to the atresia plate or to surrounding structures, they should be removed. In our study, $37.8 \%$ of patients had a malleus-incus block. We removed this atresia plate. The malleus and incus may be fixed postoperatively by fibrosis, which can be prevented by decreasing bleeding.

2) Inner ear: Touching the maleus during the drilling of the atresia plate causes high frequency losses. An iatrogenic fistula at the semicircular canals or stapes footplate may result in a dead labyrinth. Any defect formed should be closed with a fascia.

3) Formation of an ossicular chain: Allografts should be avoided, since there is a high risk of foreign body reaction. The malleus and incus may be replaced on the stapes, or a graft may just be laid directly on the stapes, forming a membranostapediopexy.

4) Graft: An annulus should be formed to insert the graft underneath, to avoid lateralization; also a skin graft should cover the fascia graft for that same purpose.

5) Eustachian tube: The function of the eustachian tube is important for hearing results.

6) EAC: Restenosis of the new ear canal and cheloids at the meatus affect hearing results. In our study $14.5 \%$ of patients had restenosis. It is related to incision technique. Use of a split thickness graft rather than a full thickness graft helps with epithelization and decreases restenosis [18].

\section{Conclusion}

EAC atresia surgery in individuals with congenital aural atresia can yield reliable, lasting hearing results with a low incidence of complications. To avoid complications, surgery should be performed by experienced surgeons. If there are coexisting abnormalities, functional results with fewer complications must be the goal.

\section{Funding}

There is no financial support or materials for this work.

\section{Conflict of Interest}

None.

\section{References}

[1] De La Cruz, A. and Teufert, K.B. (2003) Congenital Aural Atresia Surgery: Long-Term Results. OtolaryngologyHead and Neck Surgery, 129, 121-127. http://dx.doi.org/10.1016/S0194-5998(03)00531-X

[2] Chanq, S.O., Choi, B.Y. and Hur, D.G. (2006) Analysis of the Long-Term Hearing Results after the Surgical Repair of 
Aural Atresia. Laryngoscope, 116, 1835-1841. http://dx.doi.org/10.1097/01.mlg.0000233703.52308.73

[3] Patel, N. and Shelton, C. (2007) The Surgical Learning Curve in Aural Atresia Surgery. Laryngoscope, 117, 67-73. http://dx.doi.org/10.1097/01.mlg.0000240163.73601.27

[4] Bellucci, R.J. (1981) Congenital Aural Malformations: Diagnosis and Treatment. Otolaryngology Clinical North America, 14, 95-124.

[5] Chandrasekhar, S.S. and De La Cruz, A. (1995) Surgery of Congenital Aural Atresia. The American Journal of Otology, 16, 713-717.

[6] Page, J.R. (1914) Congenital Bilateral Microtia with Total Osseous Atresia of the External Auditory Canals: Operation and Report Cases. Transactions of the American Otological Society Journal, 13, 376-390.

[7] Shih, L. and Crabtree, J.A. (1993) Long-Term Surgical Results for Congenital Aural Atresia. Laryngoscope, 103, 1097-1102. http://dx.doi.org/10.1288/00005537-199310000-00004

[8] Lambert, P.R. (1988) Major Congenital Ear Malformations: Surgical Management and Results. Annals of Otology, Rhinology, and Laryngology, 97, 641-649.

[9] Lambert, P.R. (1998) Congenital Aural Atresia: Stability of Surgical Results. Laryngoscope, 108, 1801-1805. http://dx.doi.org/10.1097/00005537-199812000-00007

[10] House, H.P. (1953) Management of Congenital Ear Canal Atresia. Laryngoscope, 63, 916-946. http://dx.doi.org/10.1288/00005537-195310000-00002

[11] Patee, G.L. (1947) An Operation to İmprove Hearing in Case of Congenital Atresia of the External Auditory Meatus. Archives of Otolaryngology, 45, 568-580. http://dx.doi.org/10.1001/archotol.1947.00690010582006

[12] Nichizaki, K., Masuda, Yu. and Karita, K. (1999) Surgical Management and Its Post-Operative Complications in Congenital Aural Atresia. Acta Otolaryngologica, 540, 42-44. http://dx.doi.org/10.1080/00016489950181189

[13] Jahrsdoerfer, R.A. and Hall, J.W. (1986) Congenital Malformations of the Ear. The American Journal of Otology, 7, 267-269.

[14] Jahrsdoerfer, R.A. (1998) External Auditory Canal Atresia. In: Lalwani, A.K. and Grundfast, K.M., Eds., Pediatric Otology and Neurotology, Lippincott-Raven, Philadelphia, 533-540.

[15] Wazen, J.J., et al. (2008) Successes and Complications of the Baha System. Otology \& Neurotology, 29, $1115-1119$. http://dx.doi.org/10.1097/MAO.0b013e318187e186

[16] Hughes, G.B. (1991) The Learning Curve in Stapes Surgery. Laryngoscope, 101, 1280-1284. http://dx.doi.org/10.1002/lary.5541011205

[17] Puls, T. (1997) Stapes Surgery: Results When Performing a Moderate Number of Stapedectomies. Acta Otolaryngologica Belgium, 51, 23-25.

[18] Teufert, K.B. and De la Cruz, A. (2004) Advances in Congenital Aural Atresia Surgery: Effects on Outcome. Otolaryngology—Head and Neck Surgery, 131, 263-270. http://dx.doi.org/10.1016/j.otohns.2004.03.006 
Scientific Research Publishing (SCIRP) is one of the largest Open Access journal publishers. It is currently publishing more than 200 open access, online, peer-reviewed journals covering a wide range of academic disciplines. SCIRP serves the worldwide academic communities and contributes to the progress and application of science with its publication.

Other selected journals from SCIRP are listed as below. Submit your manuscript to us via either submit@scirp.org or Online Submission Portal.
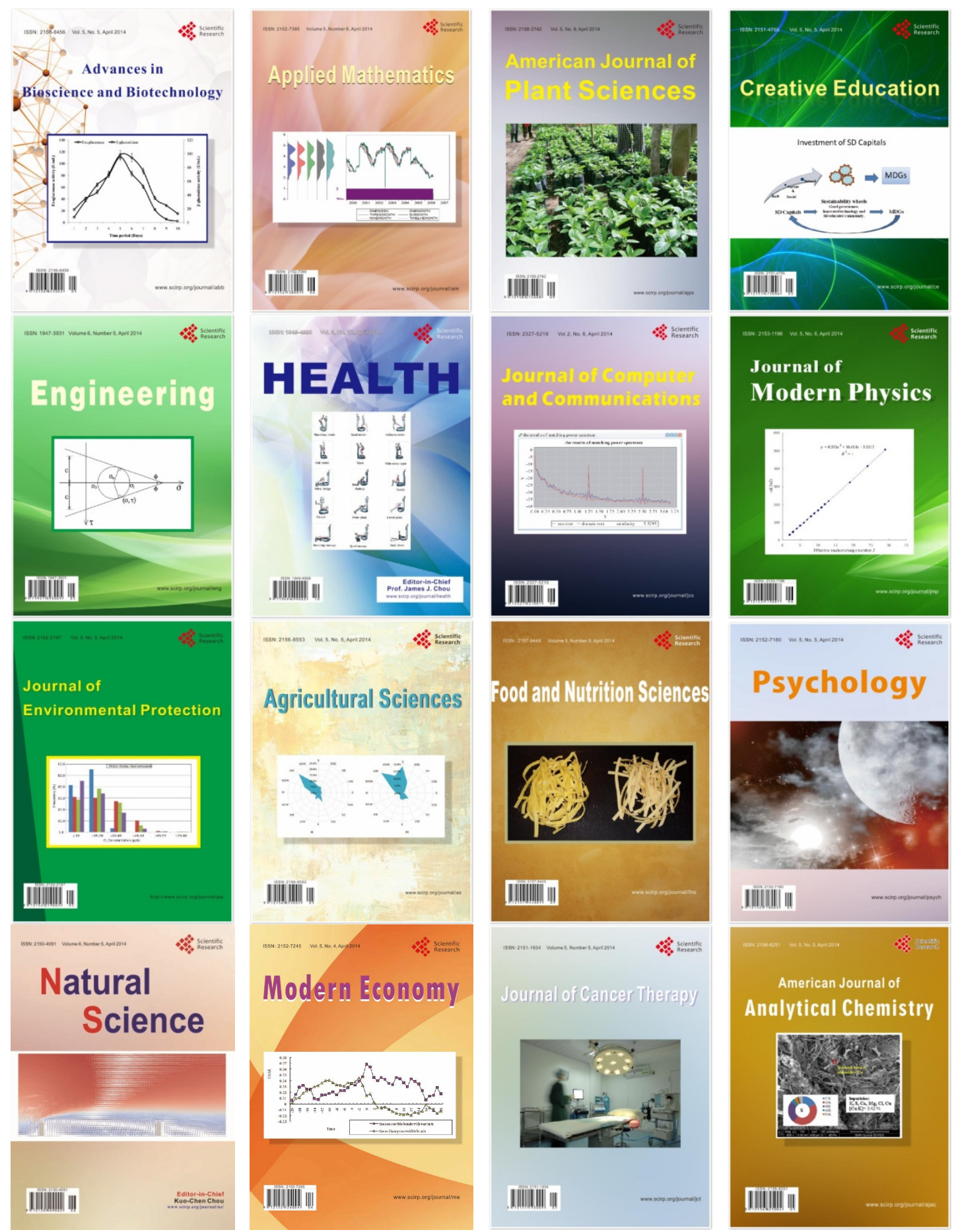